\title{
Reflets
}

Revue ontaroise d'intervention sociale et communautaire

\section{Visibilité des femmes et partenariats}

\section{Marie-Luce Garceau, Dyane Adam, Jocelyne Lamour et Caroline Andrew}

Volume 3, numéro 2, automne 1997

Visibles et Partenaires : Pratiques et recherches féministes

URI : https://id.erudit.org/iderudit/026171ar

DOI : https://doi.org/10.7202/026171ar

Aller au sommaire du numéro

Éditeur(s)

Reflets : Revue ontaroise d'intervention sociale et communautaire

ISSN

1203-4576 (imprimé)

1712-8498 (numérique)

Découvrir la revue

Citer cet article

Garceau, M.-L., Adam, D., Lamour, J. \& Andrew, C. (1997). Visibilité des femmes et partenariats. Reflets, 3(2), 23-48. https://doi.org/10.7202/026171ar

Tous droits réservés (C) Reflets : Revue ontaroise d'intervention sociale et communautaire, 1997
Ce document est protégé par la loi sur le droit d'auteur. L'utilisation des services d’Érudit (y compris la reproduction) est assujettie à sa politique d'utilisation que vous pouvez consulter en ligne.

https://apropos.erudit.org/fr/usagers/politique-dutilisation/ 


\section{Entrevue}

Marie-Luce Garceau, Sudbury

Dyane Adam, Toronto

Jocelyne Lamoureux, Montréal

Caroline Andrew, Ottawa 


\title{
Visibilité des femmes et partenariats
}

Le colloque Visibles et Partenaires comportait deux volets. Le premier portait sur la visibilité des femmes dans la société franco-ontarienne. Le second abordait le partenariat, sa définition étant appuyée d'exemples concrets et de ses enjeux. Dans l'esprit des organisatrices du colloque, ces deux aspects allaient de pair. Il faut d'abord être reconnue, prendre sa place comme femmes avant d'envisager la formation de partenariats sur une base égalitaire avec d'autres groupes, voire avec des institutions bien établies. Cette liaison allait de soi pour les organisatrices. Toutefois, ces dernières n'ignoraient pas que dans le champ de l'intervention, ces deux dimensions se révélaient plus problématiques, malgré leur criante actualité en cette époque de désengagement étatique. C'est pourquoi elles ont demandé à quatre conférencières de commenter librement sur le thème de Visibles et Partenaires.

Ainsi, au lieu de l'entrevue habituelle qu'on retrouve dans chacun des numéros de la revue Reflets, nous vous proposons les conférences d'ouverture de Marie-Luce Garceau, de Dyane Adam, de Jocelyne Lamoureux et de Caroline Andrew. Tout comme l'entrevue, ces conférences illustreront, chacune à leur manière, l'actualité du thème.

Le montage de ces conférences a été effectué par Marie-Luce Garceau, professeure, École de service social, Université Laurentienne et présidente du colloque Visibles et Partenaires.

\section{Visibles, partenaires et beaucoup plus!}

\author{
Marie-Luce Garceau \\ Professeure, École de service social, Université Laurentienne
}

Visibles et Partenaires est un colloque sur les pratiques et les recherches féministes en milieu minoritaire. Pourquoi avoir choisi ce thème qui n'est pas nouveau en soi? En effet, les médias nous bombardent constamment d'informations sur ce maitre-mot des 
années 1990 - le partenariat - qui annonce tout autant de nouvelles alliances entre industries, financiers et gouvernements que des nouvelles formes de relations entre le secteur communautaire et l'État. Face à ce vent de changement, il importait de ne pas manquer le bateau et de se pencher sur le sujet ${ }^{1}$. Pour ce faire, nous avons regroupé les femmes de l'Ontario français pour faire le point sur leurs pratiques et leurs recherches afin de développer des stratégies propres à soutenir leurs actions et à faire la promotion de changements sociaux dans ces temps de transformations.

Il est impératif de rendre visibles les pratiques et les recherches féministes alors même que nos gouvernants ont tendance à les oublier. Pour s'en convaincre, on se souviendra que lors de la dernière campagne électorale fédérale, le Premier Ministre Jean Chrétien et plusieurs chefs de partis politiques ont refusé l'invitation du Conseil du statut de la femme à venir présenter leurs plates-formes politiques sur la situation des femmes. Il importe aussi de parler de partenariat pour contrer l'indifférence de nos gouvernements à l'égard des femmes et recréer une solidarité garante de notre avenir collectif. Mais avant d'aller plus loin dans l'exploration du thème, reportons-nous en arrière.

\section{Février 1992 à Sudbury}

Plusieurs personnes se souviendront du premier colloque sur l'intervention féministe dans le Nord-Est de l'Ontario qui avait pour thème Relevons le défi. À l'époque, le Collectif des femmes francophones du Nord-Est ontarien avait choisi de faire la différence, de saisir et de fixer dans le temps, pour une première fois, notre savoir collectif (Adam 1992), notre savoir connecté (Dallaire 1992) par rapport aux interventions féministes qui se pratiquaient à la grandeur de la province. Le colloque Relevons le défi a permis de dresser un premier portrait des pratiques, des théories, des modèles qui répondaient aux besoins des femmes de l'Ontario français (Garceau 1992).

Ce rassemblement a aussi été un moment privilégié pour prendre conscience de la volonté réelle des femmes de partager 
et d'échanger sur leurs pratiques féministes, mais plus encore, de travailler individuellement et surtout collectivement à l'amélioration de la qualité de vie des femmes. En 1992, nous sommes entrées dans une démarche collective, nous nourrissant à même les multiples réalités et les différents vécus des femmes, ce qui fait encore aujourd'hui l'originalité du mouvement des femmes de l'Ontario français.

Cette démarche collective n'a jamais été délaissée malgré les difficultés et les embûches. Nous n'avons qu'à penser à la Table féministe francophone de concertation provinciale de l'Ontario, née lors du Colloque de 1992, au Réseau des chercheures féministes de l'Ontario français, à l'Action ontarienne contre la violence faite aux femmes, au Collectif des femmes francophones du Nord-Est ontarien et aux initiatives de plusieurs autres organisations, pour voir toute la vitalité et l'engagement féministes profondément enracinés dans l'action des groupes de femmes de l'Ontario français. Sans faire un bilan définitif du colloque Relevons le défi et de ses retombées pour le mouvement des femmes de l'Ontario français, un certain nombre de constats s'imposent.

Le premier est l'importance d'adopter une approche féministe dans les recherches et les pratiques touchant la situation des femmes afin de développer un potentiel libérateur qui nous appartienne, qui nous représente comme Franco-Ontariennes. Le second souligne la nécessité d'entreprendre des actions collectives afin que les femmes, les groupes et les organisations effectuent des changements sociaux radicaux dans tous les domaines de la vie sociale afin de favoriser leur épanouissement et leur autonomie. Le troisième constat affirme l'importance du combat en vue d'améliorer les conditions de vie des Franco-Ontariennes en s'appuyant sur les valeurs fondamentales que sont la solidarité, la complicité et l'entraide, et en créant de nouvelles alliances et collaborations entre les femmes et les groupes de femmes. Finalement, le quatrième constat est qu'il nous reste encore beaucoup à faire pour occuper un espace plus que symbolique dans la société, un espace concret et égalitaire, et cela, malgré les acquis des dernières décennies. 
Depuis 1992, les femmes de l'Ontario français ont maintenu l'élan, souvent jusqu'à l'épuisement, pour obtenir les changements nécessaires à une meilleure qualité de vie pour les FrancoOntariennes. Dans tous les domaines, elles ont contribué sous la bannière d'organismes locaux, provinciaux ou nationaux, sous celle d'institutions ou par leur participation à des colloques ou conférences, à obtenir des transformations. C'est donc dans le but de faire le point sur les pratiques et les recherches féministes, dans le but de soutenir les efforts de diffusion des savoirs et des pratiques des femmes que nous sommes réunies aujourd'hui.

\section{Pourquoi nous rassembler?}

Pourquoi se rassembler pour discuter de partenariat entre intervenantes, chercheures et femmes qui oeuvrent au sein d'institutions ou d'organismes sociaux, de santé et communautaires? De par leurs pratiques et leurs recherches, les féministes de l'Ontario français dénoncent toutes les formes de discrimination qui s'exercent à l'égard des femmes. Ce qu'elles revendiquent au quotidien, c'est la liberté, le respect, l'équité et l'égalité. Ce que veulent les femmes, c'est qu'elles soient non seulement visibles mais reconnues à part entière, comme des partenaires ayant droit de cité et d'être écoutées.

Si l'objectif est d'atteindre l'égalité et l'équité, les femmes sont quotidiennement confrontées à l'inégalité qui prend différents visages: pauvreté, violence, racisme, discrimination au travail, harcèlement, situation financière précaire, etc. ${ }^{2}$ À l'heure actuelle, quiconque oeuvre dans les secteurs formels et informels de la vie sociale constate rapidement que la situation vécue par les femmes est toujours fragile.

En cette ère de mondialisation, de restructuration, de privatisation, de coupures dans les services de santé, sociaux et communautaires, la vie des femmes est jalonnée de conditions difficiles. Depuis quelques années, on assiste à une baisse importante des services, résultat des compressions budgétaires gouvernementales dans divers domaines ${ }^{3}$. Celles-ci touchent directement 
les femmes et les services auxquels elles ont droit. Ce n'est pas seulement comme consommatrices que les femmes sont touchées, mais bien aussi dans leurs rôles de productrices de ces mêmes services. En effet, l'instauration et l'institutionnalisation de ces services par les gouvernements - que les femmes faisaient jadis dans l'ombre de leur foyer ou dans le privé - ont contribué largement à l'insertion des femmes sur le marché du travail et à l'obtention d'une certaine reconnaissance publique de leur travail. Or, face à de telles compressions, on est en droit de se demander ce qu'il adviendra de cette reconnaissance. De plus, lorsque ces coupures ne signifient pas l'abandon pur et simple d'un service direct offert à la population, elles contraignent souvent les organismes de femmes oeuvrant dans le secteur communautaire à une gestion financière précaire avec les conséquences que cette gestion entraine pour les intervenantes ${ }^{4}$.

Les gouvernements ontarien et canadien ne sont plus, à l'heure actuelle, des interlocuteurs privilégiés. Comme le soulignait Lucie Bélanger :

Nos gouvernements et leurs dirigeants ne se donnent plus comme autrefois la responsabilité de garantir les conditions nécessaires à l'exercice des droits reconnus. Tout au plus assurent-ils le minimum vital. L'État providence, du moins tel que nous l'avons connu, se désengage comme moteur de la création d'espaces démocratiques pour les femmes 5 .

Les coupures et les changements dans les politiques sociales qui ont cours actuellement en Ontario, ont comme conséquence l'accentuation de la dépendance des femmes et de leur pauvreté.

Si les femmes avaient fait des conquêtes, celles-ci sont aujourd'hui menacées ou ne sont plus maintenues au même niveau. D'un côté, le gouvernement conservateur et ses dirigeants cherchent à se faire rassurant en nous disant:Voyons mesdames, arrêtez de vous alarmer, vous n'êtes pas si mal tout compte fait! D'un autre côté, les femmes crient haut et fort pour que cesse le ressac, le retour en arrière. Nous écoutent-ils vraiment? 
Dans ce contexte, les actions des femmes, qu'elles oeuvrent aux niveaux de la pratique, de la recherche ou de tous autres secteurs de la vie sociale et communautaire, acquièrent une dimension stratégique indéniable en Ontario français. Les transformations contextuelles nous obligent à renouveler nos stratégies, à penser en terme de coalitions, de partenariats. Il est important d'amorcer cette réflexion, alors que les acquis des femmes sont remis en question, afin de continuer à développer des stratégies pour maintenir l'action, pour promouvoir des changements sociaux et pour en arriver à une mobilisation efficace des groupes de femmes.

\section{Deux succès franco-ontariens}

\section{Dyane Adam}

Principale, Collège Glendon, Université York, Toronto

Le thème de ce colloque Visibles et Partenaires pourrait être le leitmotiv de deux réussites franco-ontariennes en matière de partenariat communautaire dont je vais brièvement vous entretenir. La première est celle de la Table féministe francophone de concertation provinciale que vous connaissez peut être mieux, l'autre est celle du Réseau franco-ontarien d'enseignement postsecondaire. Ces deux expériences illustrent fort bien que peu importe le pavillon que l'on défende - féminisme, francophonie, lesbianisme, éducation, etc. - on ne parvient à prendre sa place et à devenir «visible» que dans la mesure où nous réussissons à être de véritables partenaires - des partenaires dans la réflexion et dans l'action.

Cette fin de semaine marque un anniversaire important pour les femmes francophones de l'Ontario. C'est ici même à Sudbury il y a cinq ans, que plus d'une cinquantaine de femmes réunies en assemblée spéciale avaient décidé de se doter d'un outil provincial de concertation et d'intervention, connu depuis sous le nom de la Table féministe francophone de concertation provinciale de 
l'Ontario. Malgré son jeune âge, la Table est aujourd'hui bien connue et respectée en province et ailleurs. Pour emprunter le thème de ce colloque, nous sommes devenus «visibles et partenaires» sur l'échiquier franco-ontarien au grand dam de certains acteurs du mouvement associatif traditionnel.

Il y a cinq ans, on ne savait pas quelle forme particulière prendrait éventuellement cette nouvelle «créature» qu'est la Table. On voulait avant tout se créer un espace commun de réflexion, de communication, de concertation et d'intervention. Pas n'importe quel espace - un lieu qui adhère le plus près possible à une vision féministe de la société où règnent les principes de justice, d'équité et de respect de la diversité des expériences de toutes les Franco-Ontariennes.

La structure de la Table est souple, les procédures minimales, les coûts d'opération allégés, les responsabilités partagées entre les bénévoles, les porte-parole multiples selon les dossiers et les expertises de chacune. Il est vrai qu'une organisation si décentralisée comporte ses risques - entre autres de fragmentation, d'éparpillement, de communications aléatoires et incomplètes mais dans l'ensemble, cet espace de leadership «transversal», pour emprunter l'expression de Lyne Bouchard, s'est avéré une importante pépinière d'idées, de projets novateurs et surtout une pépinière de sororité amicale et solidaire. La nature des rapports entre les membres, la façon de mener nos dossiers et d'agir sont aussi sinon plus importantes que les résultats eux-mêmes. Je ne veux pas donner l'impression ici que la Table est à l'abri des conflits internes, des tiraillements et des rivalités propres à tout groupement humain. Nous ne le sommes pas, mais les mésententes et les différends font, dans la mesure du possible, l'objet de discussions ouvertes, franches et parfois douloureuses.

Il y a quelques semaines, je lisais un article dans le Globe and Mail qui s'intitulait "Women without men» de la chroniqueure Margaret Wente. J'en suis venue à la conclusion que de tous les groupes auxquels je participe en ce moment, c'est certes la Table qui reproduit le plus fidèlement la structure sociale des bonobos. Et croyez moi, vous aimeriez vivre, du moins à certains égards, comme les bonobos. 
Les bonobos sont une espèce de primates peu connue qui vit dans la jungle du Zaïre et - mesdames - où la femelle et non le mâle est en charge de la tribu. La structure sociale des bonobos est maintenue par des alliances bienveillantes entre les femelles adultes. Elles se tiennent entre elles quand les mâles ont des écarts de conduite. Les femelles ne permettent aucun "cognage de torse», ni chuintement, ni crissement de dents ou tout autre comportement d'intimidation et de violence chez les mâles. Les bonobos sont pacifiques, égalitaires et sociables. Ce sont les femelles qui veillent à maintenir des rapports harmonieux entre les membres de la tribu. Comme chez le genre humain féminin, les femelles semblent avoir une orientation pour la solidarité et la cohésion (connectedness). Bien sûr, les bonobos n'ont pas le don de la parole et elles ont recours à d'autres moyens pour maintenir la cohésion et l'harmonie entre elles et avec les mâles. Pour manifester leur affection réciproque et leurs tendances amicales, les bonobos s'engagent constamment dans des rapports sexuels - hétéro, homo, intergénérationnels. Bref, la sexualité sous toutes ses formes est mise au service de la paix sociale et de la solidarité entre ces primates. Ce n'est pas la force physique, l'agressivité et le contrôle d'autrui qui conditionnent les rapports entre eux, mais la sexualité et la tendresse.

Je suis convaincue que la grande majorité de nos concitoyens et concitoyennes trouveraient ce genre d'organisation sociale aux moeurs sexuelles libérales tout à fait dépravée et vouée à la perdition. En revanche, on n'est pas particulièrement troublé par la violence conjugale, l'abus des enfants, les agressions sexuelles et la criminalité et on accepte bien de vivre dans une telle société. La morale de cette histoire est la suivante. Sans nécessairement encenser les pratiques particulières de cohésion des bonobos, je nous souhaite grand succès à revendiquer et à imposer une société fidèle à la structure «bonobienne». En cela, la Table a une bonne longueur d'avance dans le milieu associatif franco-ontarien!

Il y a tout de même d'autres efforts de concertation et de réseautage dans la communauté franco-ontarienne qui s'inscrivent à une vision similaire de partenariat et de réseautage et qui, comme la Table, aboutissent à des résultats remarquables et bien visibles. 
En fait, cela fera bientôt une décennie que je suis active en administration universitaire.Au cours de cette période, j'ai travaillé à de nombreux dossiers et projets. Mais une des réalisations dont je suis bien fière, c'est d'avoir été l'une des artisanes du Réseau franco-ontarien d'enseignement à distance (le Réseau). Le Réseau a un volet collégial et un volet universitaire. Je vais me limiter à parler de ce que je connais le mieux - le réseau universitaire et à souligner son importance pour la communauté des femmes francophones de l'Ontario.

On a tendance à voir le Réseau d'abord et avant tout comme une infrastructure de communication technologique mais c'est beaucoup plus que cela. Le Réseau, c'est un rêve, c'est une vision en matière de formation universitaire en français pour l'Ontario. À plusieurs égards, les établissements d'enseignement postsecondaire ont les mêmes défis que la communauté dans leurs efforts de servir leur population: dispersion et isolement géographiques de la clientèle, faible poids démographique, rareté des ressources humaines et financières, inégalité régionale des services, concurrence avec les établissements anglophones. Plutôt que de continuer à travailler de façon indépendante et isolée, avec des ressources limitées et souvent en concurrence entre elles, les sept universités bilingues de la province (Université d'Ottawa, Université St-Paul, Université Laurentienne, Collège universitaire de Hearst, Université de Sudbury, Collège dominicain, Collège universitaire Glendon de l'Université York) ont choisi en 1994 de se regrouper en consortium pour planifier conjointement, au niveau provincial, les services universitaires en français et élargir, dans la mesure du possible, la gamme des cours et des programmes d'études offerts en français dans chacune des régions où on retrouve une concentration importante de francophones.

- Les universités se sont d'abord entendues sur le transfert complet de crédits d'une université à une autre. Par exemple, un cours d'introduction en psychologie suivi à Hearst ou par correspondance à l'Université Laurentienne serait reconnu pleinement par les autres universités du réseau. Par le fait même, la mobilité des étudiantes et des étudiants s'en trouvait nettement améliorée. Pour des étudiantes à temps partiel, ceci 
signifie que leur choix de cours ne se limite plus à ceux offerts par l'université dans laquelle elles sont inscrites, mais qu'elles peuvent accumuler des crédits dans d'autres universités.

- Les universités se sont dotées de la même infrastructure technologique pour favoriser l'échange de cours et de programmes d'une région à une autre - plus précisément, la création d'un réseau de salles de classe vidéointeractives jointes par des réseaux télématiques qui compte actuellement plus d'une trentaine de sites répartis dans 15 localités, elles-mêmes dispersées sur l'ensemble du territoire ontarien. On cherche à rejoindre ainsi la clientèle étudiante dans sa localité plutôt que d'attendre qu'elle se rende sur le campus - un plus pour les femmes qui sont de loin le groupe le plus important parmi la clientèle étudiante inscrite à des études à temps partiel et à distance.

- Les universités ont développé conjointement un plan d'enrichissement de leurs programmes d'études respectifs par l'échange régulier de cours. Ce plan couvre plus d'une quinzaine de disciplines dans les humanités, les sciences sociales et les sciences.

- Les universités comptent également développer de nouveaux programmes-réseau dans des secteurs spécifiques comme les sciences de la santé. J'aimerais mentionner ici le projet d'un programme-réseau en études féministes pour l'Ontario français. C'est un projet que caressent depuis longtemps certaines universitaires et le Réseau des chercheures féministes de l'Ontario français. Le tout récent appui donné par le MÉF au Consortium des universités de la francophonie ontarienne pour le développement des programmes-réseau de baccalauréat et de maitrise en études des femmes permet de croire qu'on pourra enfin réaliser ce projet scolaire. Outre la formation de diplômées en études féministes, on souhaite que cette entreprise-réseau débouche sur des projets de recherche sur les femmes francophones vivant en milieu minoritaire et en collaboration avec ces dernières. Et pourquoi cette entreprise réseau ne pourrait-elle pas devenir les assises d'un partenariat plus étroit et continu avec la communauté des femmes 
francophones de l'Ontario - un partenariat qui vise la formation, la recherche, l'organisation de colloques, la diffusion et la publication d'ouvrages qui documentent la réalité des femmes francophones vivant en milieu minoritaire.

- Les universités et les collèges communautaires - membres du Réseau d'enseignement à distance - ont également convenu que durant les périodes où le réseau technologique ne sera pas utilisé à des fins d'enseignement et de rencontres interinstitutionnelles (fins de semaine, soirs, etc.), qu'il soit mis au service des différents organismes communautaires tels que la Table et ses différentes unités constituantes qui voudraient s'en servir pour leurs propres rencontres, colloques ou activités de formation.

Voici donc quelques réalisations d'un effort de concertation et de collaboration du secteur universitaire de l'Ontario français pour mieux servir sa communauté. Aboutir à une telle concertation interinstitutionnelle a exigé un important changement de paradigme par rapport à la façon dont chaque établissement concevait sa planification scolaire et la gestion de ses ressources jusqu'à présent. Par exemple, l'évolution vers un plan provincial d'offre de cours et de programmes en français, fondé sur un important flot d'échanges interinstitutionnels, exige qu'on subordonne, jusqu'à un certain point, le contenu de la programmation - sinon le contenu même des programmes et peut-être même le recrutement professoral - aux résultats d'une concertation avec les établissements partenaires. À l'intérieur même des universités bilingues, et sans qu'aucune décision spectaculaire ne soit prise, une sorte d' «université sans mur» francophone est en train d'émerger en Ontario et ceci grâce à une concertation et une volonté collectives de la part de tous les partenaires en éducation universitaire. Quand on connait la valeur sacro-sainte que revêt l'autonomie institutionnelle dans le monde universitaire, on est plus en mesure d'apprécier le pas énorme des universités bilingues en vue de créer un système cohérent et concerté de formation universitaire en français dans cette province.

En guise de conclusion, rappelons qu'au même moment où les groupes de femmes s'organisaient autour de la Table et que le 
Réseau franco-ontarien d'enseignement à distance et le Consortium des universités de la francophonie se consolidaient, d'autres secteurs de l'Ontario français se dotaient également pour la première fois de mécanismes provinciaux de réseautage. Mentionnons entre autres le Regroupement des intervenantes et intervenants francophones en santé et services sociaux de l'Ontario (RIFSSSO) créé en 1994, dont l'objet est de promouvoir la communication entre les membres et de les aider à améliorer la prestation des services en français en Ontario, et d'autres tel le Réseau des chercheures féministes de l'Ontario français. Cette effervescence de nouveaux regroupements provinciaux en Ontario français marque un mouvement fondamental dans le leadership traditionnel de la communauté. Bien que l'ACFO ait récemment réussi, grâce à l'appui des instances fédérales, à maintenir son statut fort contesté de porte-parole officiel de la communauté francophone de l'Ontario, la base de son leadership demeure bien précaire. À mon avis, il ne tardera pas que d'autres regroupements se joignent à la Table pour réclamer un nouveau leadership en Ontario français qui incorpore la diversité. Il est à souhaiter que cette dernière en devienne sa signature particulière.

\section{Éléments de réflexion sur le thème Visibles et Partenaires}

Jocelyne Lamoureux

Professeure, Université du Québec à Montréal

Je voudrais d'abord remercier très sincèrement les instigatrices du colloque Visibles et Partenaires, de m'avoir invité à cet événement. Cette invitation m'a surprise, ravie, et je dois aussi l'avouer, rendue un peu confuse. Je me questionnais sur ma légitimité de prendre la parole alors que les rangs du mouvement des femmes et, plus particulièrement, ceux du mouvement féministe en Ontario francophone, sont si bien pourvus d'intervenantes exceptionnelles, de 
pionnières, de participantes d'expérience et de chercheures chevronnées.

Je me suis souvenue qu'au cours des dernières années, le mouvement des femmes de l'Ontario francophone s'était ouvert à la diversité culturelle. Entre autres, le colloque Sensibiliser, décider et agir qui s'est tenu à Ottawa en 1994, avait permis toute une réflexion, que je considère importante, visant l'inclusion des mécanismes et le fonctionnement des rapports sociaux de sexes dans une redéfinition des rapports ethnicité-féminité. Des débats actions, explorant le thème d'une culture féministe universelle et plurielle capable d'intégrer des différences au chapitre des sexes, des classes, des ethnies, des régions, et je l'imagine, des nations, étaient à l'ordre du jour. Comme je vis maintenant au Québec,je me suis alors dis : «C'est peut-être pour cela qu'elles m'ont invitée». Après coup, je me suis dit: «Elles m'ont peut-être invitée parce que je suis Franco-Ontarienne de naissance», alors je vais en profiter.

Ainsi donc, je tiens par dessus tout à saluer la passion de l'action qui caractérise les femmes de l'Ontario français. J'en sais quelque chose parce que j'en ai «souffert» un peu dans ma jeunesse et au moment de mon adolescence alors qu'éclatait la culture rock-nroll. Non, ma mère n'était pas féministe, mais ma grand-mère s'était battue avec des aiguilles à chapeau contre les policiers à cheval de l'Ontario Provincial Police, au moment du règlement 17. Et jusqu'à l'âge de 14 ou 15 ans, je n'ai pas pu écouter la radio en anglais parce qu'il fallait "protéger notre langue». Alors, du fin fond de mon enfance et de mon adolescence, j'ai connu la vigueur et la passion des femmes, entre autres celle de ma grand-mère, pour la cause non seulement des femmes, mais aussi des francophones en Ontario.

J'aimerais partager avec vous quelques réflexions sur le thème Visibles et Partenaires. Au premier abord, dans toute sa positivité, de quoi parle-t-on lorsque l'on évoque la visibilité? Qu'est-ce que ce mot d'ordre implique? Il exprime la parole, la "parlure», la couleur, l'expressivité, l'éclat, le rayonnement, le dérangement, la radicalité. Penchons-nous un instant sur la question d'être radicale ou non. Vous savez que le mot radical, au sens étymologique du 
terme, signifie «aller à la source, à la racine» et je pense que nous tentons toutes d'aller à la source de ce qui fait mal. À ce momentlà, nous sommes toutes des radicales, qu'on le veuille ou non.

Quant au terme partenariat, il implique évidemment la complicité, les projets, les partages, les engagements communs. C'est bien stimulant, c'est festif, c'est réjouissant aussi, et c'est bien qu'il en soit ainsi. En effet, il semblerait, sans en faire évidemment quelque chose de biologique, que c'est une manière de faire qui nous est propre, à nous les femmes. Actuellement, je travaille avec des collègues sur la culture organisationnelle des groupes de femmes, et cette façon d'être ensemble apparaît effectivement assez importante.

Mais le thème Visibles et Partenaires soulève aussi une question grave, pleine d'enjeux, de défis et d'embûches. Que signifie la notion de visibilité? Au fond, il s'agit d'un certain rapport au monde. Qu'il suffise de penser au fait que le droit de vote n'est pas acquis pour les femmes dans certaines régions du monde ou encore que de grandes initiatives sont prises par les gouvernements et les Églises pour statuer sur le vivre ensemble ou la loi, et nous n'y serons pas! Dans un autre ordre, notre travail de production et de reproduction de la vie au jour le jour n'est ni comptabilisé, ni reconnu; il est donc invisible et innommable!

Ainsi, le mot visible implique une exigence, celle d'avoir un autre rapport au monde, et cela évoque nos luttes. Quand des associations telles l'Association des fermières de l'Ontario ou l'Union culturelle des Franco-Ontariennes rappellent le savoirfaire des mères sur toutes les tribunes, elles disent haut et fort que celui-ci nous donne le devoir et le droit de participer à la vie publique. Quand, au Québec,l'Association féminine d'éducation et d'action sociale (AFÉAS) présente son mémoire à la commission Bird, dans les années 1960, elle n'affirme qu'une seule chose: que toutes les femmes travaillent. Donc, sortir le travail des femmes de l'invisibilité a été un grand dossier pour ces associations.

Par ailleurs, quand les femmes des groupes communautaires de l'Ontario ont tenté de rendre visible la violence faite aux femmes et de briser le silence qui l'entoure, aux moyens d'assemblées, de colloques, de rencontres, de kiosques, de vidéos, cela 
évoque aussi, pour moi, le thème de la visibilité. En effet,la visibilité implique rompre le silence. À ce propos,je me rappelle que l'étude de besoins portant sur la création des services en français pour les survivantes d'agressions à caractère sexuel s'intitulait justement Rendre visible, l'invisible. Alors, vous comprendrez que cette thématique est récurrente. Elle revient sans cesse et illustre notre rapport au monde et nos luttes.

J'aimerais poursuivre cette exploration du thème de la visibilité et d'un autre rapport au monde à l'aide de deux autres exemples. En 1979, la Fédération des femmes canadiennes-françaises présente un rapport qui aborde les préoccupations des femmes pour leur santé et celle de leur famille, rapport qui, d'une part cherche à faire le point sur leur groupe en vue de développer leur compétence en organisation communautaire et en action politique et, d'autre part qui vise à sortir les femmes de l'isolement. Vous vous souviendrez peut être de ce rapport intitulé Valoriser et faire reconnaitre la part des femmes. Il s'agit donc, encore une fois, de faire émerger les femmes à la visibilité. Par ailleurs, j'assistais récemment, à Montréal, à la remise d'un prix nommé La tête haute, de l'Association pour la défense des droits des travailleuses domestiques. Ce prix signale l'action d'une travailleuse domestique: ce qu'on appelait jadis les «bonnes». Donc, lorsqu'une travailleuse domestique des Philippines s'est présentée à la tribune pour recevoir son prix, elle a dit: «j'ai un rêve».J'ai pensé tout de suite à la célèbre expression de Martin Luther King: "I Have a Dream». Par la suite, elle a ajouté «j'aimerais être visible». En effet, les travailleuses domestiques ne sont pas inclues dans les normes minimales de travail. Elles sont invisibles, elles n'existent pas. On peut les considérer comme les dernières esclaves d'un système qui n'en finit plus de mourir.Ainsi, la demande de visibilité traduit une demande de reconnaissance et d'existence pour les femmes et leur mouvement.

De plus, j'ajouterais que pour le mouvement des femmes en Ontario auquel vous appartenez, l'autre rapport au monde se conjugue avec des revendications ayant trait à l'appartenance ethnique. Lier de concert l'appartenance ethnique et le féminisme c'est, non seulement, comme le dit la chercheure Linda Cardinal, 
faire en sorte que les femmes ne soient pas déterminées uniquement par des rapports sociaux de domination (de rapport de sexage), mais qu'elles participent aussi à la création d'une vision du monde dans et par laquelle elles tentent d'émerger comme sujet $^{6}$. Or, cette façon d'être au monde qui anime le mouvement des femmes en Ontario, ce questionnement féministe des milieux francophones minoritaires pourrait et devrait également devenir plus visible au sein des discours et des pratiques du mouvement des femmes au Canada et au Québec. Je vais y revenir.

Pour l'instant, je voudrais examiner la thématique de la visibilité en lien avec le travail de recherche. Je m'intéresse surtout aux projets de recherche qui donnent la parole aux femmes et qui visent à redonner un poids d'existence concret «aux exclus du regard et de la pensée ou encore de la parole et de l'action", comme le dit la chercheure Ellen Corin ${ }^{7}$. À travers les recherches, le fait de donner ainsi la parole aux femmes leur permet de retrouver une densité en rendant les univers de sens et d'action auxquels elles participent aussi visibles.

J'ai donné plusieurs exemples de ce que signifie devenir visibles par rapport à nos vies, à nos mouvements et à nos luttes. Or, devenir visibles implique de constater que nous sommes, non seulement nombreuses, mais aussi que nous sommes diverses et multiples avec nos systèmes de sens, de signes et d'actions plurielles. Cet aspect m'apparaît essentiel. Linda Cardinal n'exprimait pas autre chose lorsqu'elle disait que le fait d'appartenir à la catégorie des femmes est consubstantiel à une grande hétérogénéité. Or, cette réalité comporte certains enjeux. En effet, lorsque les femmes se révèlent, lorsqu'elles deviennent visibles et lorsqu'elles s'aperçoivent qu'elles sont multiples, qu'est-ce que ça implique?

D'abord, ça signifie qu'il faut se poser des questions difficiles auxquelles on a malheureusement répondu historiquement de façon trop simple. On y a répondu en disant: «Nous sommes des soeurs, nous vivons la sororité». On a dit: «Nous sommes représentées sur le mode de la communauté, fondée en grande partie, évidemment, sur la communalité de notre statut de victime ou d'assujettissement». On a insisté sur l'expérience commune des femmes, sur le féminin comme une carte d'identité. Un tel 
discours féministe nous a placé dans l'idée d'une classe de sexe, d'une certaine homogénéité de la catégorie sociale «femme».

Or, ce positionnement doit être discuté, doit être remis en question. Les femmes doivent avoir le courage de se poser des questions parce que ce positionnement uniforme, communautaire dans le sens de "partager une identité commune», pose un problème.

En tous cas, il en a posé aux lesbiennes du mouvement des femmes qui ne se sont pas senties à l'aise dans cet espace un peu totalisant. Il en a posé aux «Black Sisters» américaines qui ont remis en cause la belle unanimité de la sororité. Il en a posé aux femmes immigrantes lors des célébrations, à Montréal, du cinquantième anniversaire du droit de vote des femmes. De plus, cette question de la pluralité de nos pratiques et de nos vies qui s'oppose à une totalité de la sororité et de l'oppression commune, continue de poser problème aux femmes acadiennes et aux femmes francophones qui vivent en milieu minoritaire. Et cela me semble très important. En revendiquant l'appartenance ethnique au sein du féminisme, celles-ci se trouvent à remettre en cause la visée homogénéisante associée à la sororité. Sororité, oui, personne ne s'y opposera, mais accueil à l'hétérogénéité, aux différences, à la totalité de l'expérience des femmes.

Je reprends ici la réflexion très importante d'une collègue de l'Université Laval à Québec, Diane Lamoureux, qui propose de se questionner sur les modes de constitution du mouvement des femmes. Non pas pour tout foutre en l'air, mais pour mettre en lumière la difficulté du mouvement à se penser en terme d'un «nous différencié» qui s'édifierait sur la base de «je» complexes. Il ne s'agit pas d'une simple addition de «je», mais de «je» complexes. Au fond, il ne s'agit pas de poursuivre la quête d'une identité commune.Admettre et n'admettre que la similitude des femmes, c'est passer sous silence que nous vivons dans des sociétés fragmentées, soumises à des logiques diverses de domination. Et l'on ne peut pas faire de l'une de ces logiques de domination, que ce soit le sexe, la classe, l'orientation sexuelle, l'ethnicité, l'âge ou quoi que ce soit d'autre, la quintessence de toutes les autres.Ainsi, la question de la fluidité des identités personnelles et sociales de 
la multiplicité des identités du sujet émerge et pose avec plus d'acuité la question du pluralisme.

Il ne s'agit pas seulement de dresser une belle tribune où l'on trouverait obligatoirement une femme ordinaire, une femme lesbienne, une femme juive francophone, une femme noire, une femme jeune, une femme âgée aux cheveux gris, etc. Il ne s'agit pas seulement de représenter la différence à une table ronde pour répondre aux exigences de la rectitude politique (politically correct $^{8}$. Il ne s'agit pas non plus d'un pluralisme passif où la différence va céder le pas à l'indifférence, mais d'un pluralisme de participation où les différences contribuent, dans leur tension même, à l'élaboration de nouveaux modèles de pratique. Ce mode, certes, sera très exigeant et bien moins rassurant que ce que nous avons coutume de vivre. Il n'est pas question de tout renier mais d'ouvrir d'autres fenêtres, de se penser dorénavant autrement. En somme, il s'agit de se donner comme objectif, comme le dit si bien Diane Lamoureux, une communauté à construire, à partir de l'expérience singulière de chacune, à partir de la fluidité de nos identités personnelles et sociales. Et si, pour cela, nous devons mettre de côté - je m'en excuse - la tentation de la «nature féminine», soit l'insistance sur notre oppression, notre expérience réduite à nos hauts faits passés, nos organisations trop étroites parfois, la forme collective de celles qui partagent nos opinions et où sont tues les différences et les dissensions, en somme, notre havre, et bien, nous devons le faire!

C'est au prix d'une réflexion en profondeur sur ce sujet difficile que nous pourrons faire une contribution magistrale au vivre ensemble où nous tenterons de repenser la culture politique autour de la notion de citoyenneté et de la lutte à l'exclusion. Ce sont là mes réflexions sur le thème de la visibilité, mais accordez-moi encore un moment sur le thème du partenariat.

Moi, je suis à la fois une sceptique et une fanatique du partenariat.Je suis une fanatique du partenariat parce que je trouve qu'il n'y a pas moyen de s'en sortir autrement. Je suis également une sceptique du partenariat, parce que je ne pense pas qu'il soit très lucide, ni très prudent, d'envisager les partenariats comme des grands "partys» où on travaille ensemble. Il faut bien voir que 
le partenariat est devenu un thème très à la mode au moment même où l'État-providence se désengageait.

L'impact des coupures est terrible. En Ontario, vous en savez quelque chose. Je ne reviendrai pas sur la litanie des ravages. Pourtant, lorsqu'on se penche sur l'évolution des politiques en matière de santé, de services sociaux ou d'éducation, et qu'on nous ramène sans cesse le thème du partenariat, je pense qu'on a un peu raison d'être sceptique. Il ne faudrait pas dire que le partenariat est dangereux, qu'il ne faut pas s'y engager. Il nous faut développer ce que j'appelle la pensée complexe et développer l'utilisation du paradoxe dans l'analyse, entre autres, des partenariats.

Pour analyser le partenariat, il faut refuser de fonctionner dans les catégories de noir ou de blanc, de bien ou de mal, de lutte ou de récupération, de l'intégration ou de la subversion. En effet, le partenariat est un rapport social extrêmement paradoxal et extrêmement complexe, et ce n'est pas en donnant des mots d'ordre de partenariat que tout ira bien.

Il faut retenir que le partenariat se résume trop souvent en une stratégie gestionnaire extrêmement coûteuse pour les mouvements sociaux, qu'il s'agisse du mouvement des femmes ou du mouvement communautaire. C'est pour cette raison qu'il faut concevoir les partenariats dans un rapport un peu plus global, les voir comme un rapport social important, incontournable certes, qui a deux caractéristiques ${ }^{9}$. Le premier attribut, c'est l'asymétrie, la différenciation, ces termes se référant au fait que les acteurs en cause ont des cultures différentes, et donc qu'il y a un choc de cultures dissemblables. Si vous êtes intervenante dans un groupe de femmes et qu'une femme se présente à votre maison d'hébergement, dans votre comité de lutte aux agressions sexuelles, dans votre centre de santé, dans votre groupe de formation, vous n'êtes pas dans un rapport d'égalité avec cette personne. Malgré tous les beaux discours, vous demeurez une intervenante reconnue pour son expertise. Si vous participez à une recherche-action avec des universitaires et que vous provenez du milieu communautaire (des groupes de femmes, des groupes de base), vous n'êtes pas en relation de savoir d'égale à égale. Si vous 
concluez un partenariat avec des institutions de la santé, des services sociaux ou de l'éducation, vous n'êtes surtout pas en rapport égalitaire, ne serait-ce qu'au plan de la gestion. Ainsi, pour moi, la première caractéristique du partenariat, c'est cet espace d'asymétrie de cultures en choc, où les acteurs en cause ont «des cultures spécifiques, avec des moyens discursifs, organisationnels et matériels différents» ${ }^{10}$. Quelquefois, ce choc est très douloureux, surtout si nous faisons fi de nos différences.

La deuxième caractéristique du partenariat, outre l'asymétrie ou la différence de culture, c'est évidemment la hiérarchie, le pouvoir inégal, soit les rapports de forces qui se nouent dans une logique de relative dépendance et d'autonomie ${ }^{11}$. Le pouvoir n'est pas toujours du même côté.Je ne pense pas qu'il y ait des opprimés économiques partout et toujours, et qu'il s'agit toujours des mêmes personnes. Il y a cependant un pouvoir inégal dans les relations sociales.

Si le partenariat présente ces deux caractéristiques, je pense que nous devrions plutôt le voir comme étant de l'ordre de la coopération conflictuelle ${ }^{12}$. Ainsi, dans un partenariat, on collabore, on coopère, on tente de s'entendre, on tente de faire des actions ensemble, mais on se garde aussi un espace de débat fort, de discussion forte. Il est faux de penser que tout doit se faire dans le style "Youp là, là, on s'aime». Ce n'est pas vrai! Cela ne se passe pas ainsi dans la vie. En ce sens, mieux vaut parler de coopération conflictuelle lorsqu'il y a des cultures en choc et des rapports hiérarchiques; nous serions alors un peu plus lucide. Certes, nous devons travailler pour que les partenariats fonctionnent, parce que dans la conjoncture actuelle, c'est un rapport social absolument essentiel qui demande de bonnes doses de pédagogie participative, pour s'apprivoiser, pour se donner des espaces d'autonomie.

Voilà,j'ai l'impression que sur la thématique de la visibilité et du partenariat, il peut y avoir des mises en garde et des défis. Il peut y avoir, évidemment, un projet commun, mais cela nous demande, je pense, beaucoup de lucidité et de courage. C'est ce que je nous souhaite. 


\section{Visibilité comme condition du partenariat}

\section{Caroline Andrew}

Professeure, science politique, Université d'Ottawa

En m'inspirant du thème du colloque Visibles et Partenaires, j'ai décidé de discuter de la visibilité comme condition du partenariat. Je développerai cette idée en me basant sur mon évolution personnelle comme chercheure et participante dans les projets de recherche que j'ai effectués en partenariat avec des groupes communautaires. Cela dit, pour débuter, j'utiliserai une image qui ne fait aucunement référence à mes propres recherches pour illustrer ma conception du partenariat. Cette image, issue d'une réflexion, m'est venue lors d'un séminaire portant sur les femmes et le développement.

Ce séminaire regroupait des chercheures canadiennes et des chercheures d'autres pays, en provenance surtout de l'Asie. Durant ce séminaire, $\mathrm{j}$ 'ai assisté à la présentation d'une chercheure vietnamienne, Oahn, qui travaillait dans le cadre d'un programme conjoint en études des femmes et en travail social, sous l'égide de l'Agence canadienne de développement international (ACDI), mais ça c'est une autre histoire.

Oahn est une femme remarquable. Elle a vécu et survécu à l'ensemble des bouleversements ayant affecté leVietnam au cours des quatre dernières décennies. Malgré tout, elle a su garder une sérénité, une sagesse et une détermination sans faille en vue d'améliorer la vie des femmes vietnamiennes et de travailler au développement de son pays.

Oahn a fait une recherche avec des cadres locaux de l'Union des femmes du Vietnam. Or, il faut préciser que l'Union des femmes est l'organe officiel des femmes, donc une organisation du Parti. La recherche participative visait à former et à outiller les cadres locaux pour qu'elles puissent travailler aux transformations culturelles affectant le rôle des femmes en général. Loin de se 
limiter uniquement aux aspects légaux et politiques, cette recherche qui s'est faite par entrevues de groupe, avait une visée plus large. Oahn a conclu sa conférence en disant que les participantes, les cadres locaux et les chercheures ont beaucoup ri, et donc, que la recherche est de bonne qualité, que l'ambiance joyeuse témoigne de la validité des données. Sur le coup, nous avons toutes ri.

Par la suite, j'ai beaucoup réfléchi à la pertinence de ces remarques de Oahn. Je crois qu'elle a profondément raison et que le rire symbolise très bien les qualités essentielles d'une bonne recherche faite en partenariat. Premièrement, le rire est d'abord l'expression d'une ambiance d'égalité et surtout de complicité. On ne rit pas avec aisance en présence d'inégaux. Rire ensemble, c'est une marque de convivialité et de partage. Le rire crée des liens de solidarité qui établissent une égalité fondamentale entre les participantes.

Deuxièmement, le rire exprime, pour moi, un bon climat d'apprentissage mutuel, une ambiance détendue où on peut poser des questions sans gêne, et, où on peut apprendre à se connaître. Travailler en partenariat implique nécessairement un apprentissage mutuel.

Troisièmement, le rire complice vient également avec la découverte. On rit parce qu'on vient de comprendre quelque chose. C'est le rire d'éclaircissement; on est contente parce qu'on voit tout à coup le monde de façon nouvelle ou différente. C'est un moment clé de la recherche et je crois qu'il est souvent exprimé par un rire.

Finalement, l'aspect fort probablement le plus important et le plus simple, le rire représente la voix des participantes. C'est une façon de briser le processus traditionnel de la recherche et de donner la voix aux participantes. Ce n'est plus l'expert ou l'experte qui interprète, ce sont les participantes qui s'expriment. Cette voix coupe la hiérarchie traditionnelle de la recherche en exigeant qu'on tienne compte de la vision de celles qu'on étudie. En réalité, cela signifie bien davantage, car on n'étudie plus, on travaille avec les participantes. Le rire, c'est le bruit qui éveille à l'autre. 
Pourquoi insister tellement sur la visibilité des partenaires? Il me semble que c'est là une condition essentielle, car traditionnellement les sujets, objets de recherche, n'ont pas été visibles, alors que les chercheurs et chercheures, eux, le sont terriblement. Alors, pour créer l'égalité qui est au fondement d'un véritable partenariat, il faut que toutes les partenaires soient également visibles.

Il faut donc trouver des façons pour permettre l'expression de cette voix authentique des partenaires et assurer que le travail des chercheures n'est pas de "faire une étude sur», mais bien de chercher ensemble le sens des événements, des processus et des actrices. Les occasions de faire de la recherche visant à rendre visibles les préoccupations, les situations et les priorités des femmes de l'Ontario français sont nombreuses et les voies qui permettront aux partenaires, surtout aux groupes de femmes et aux groupes communautaires, d'être visibles le sont tout autant. Toutefois, il appartient à chaque groupe de réfléchir sur les moyens qui lui conviennent pour réaliser cette visibilité, compte tenu des conditions spécifiques de leurs ressources et de leurs stratégies d'action. Mais, pour réussir à donner l'orientation que vous voulez collectivement donner à votre recherche, il va falloir que les groupes de femmes, les chercheures universitaires, les chercheures autonomes et les organismes subventionnaires travaillent en partenariat. Il faut donc explorer des façons innovatrices pour réaliser ce défi, pour comprendre la réalité complexe et mouvante des femmes canadiennes et pour faciliter la formulation des politiques et des programmes dont l'objectif est d'améliorer les réalités auxquelles les femmes sont confrontées. Ce sont des partenariats importants et, nous l'espérons, d'autres occasions de rire ensemble.

\section{Bibliographie}

ADAM, Dyane (1992). «Préface», dans Marie-Luce Garceau (dir. de publ.), Relevons le défi! Actes du colloque sur l'intervention féministe dans le Nord-Est de l'Ontario, Ottawa, Les Presses de l'Université d'Ottawa, coll. Actexpress.

DALLAIRE, Hélène (1992). «Un Savoir connecté», dans Marie-Luce Garceau (dir. de publ.), Relevons le défi! Actes du colloque sur l'intervention féministe dans le Nord-Est de l'Ontario, Ottawa, Les Presses de l'Université d'Ottawa, coll. Actexpress, 37-44. 
GARCEAU, Marie-Luce (dir. de publ.) (1992). Relevons le défi! Actes du colloque sur l'intervention féministe dans le Nord-Est de l'Ontario, Ottawa, Les Presses de l'Université d'Ottawa, coll.Actexpress.

\section{Notes}

1. Dans ce texte d'ouverture, il ne s'agit pas d'explorer le sens des thèmes principaux du colloque, d'autres conférencières en parleront abondamment. Plutôt, il s'agit de présenter le contexte dans lequel ces thèmes sont apparus et l'origine de notre questionnement.

2. Voir à ce sujet la Déclaration des femmes de l'Ontario du 6 décembre 1995, publiée dans C. Godin et G. Sirois, (1996), «La violence faite aux femmes: analyse de la situation en Ontario français», Reflets, vol. 2, no 1,116-133.

3. On pense ici aux programmes de santé, sociaux et communautaires, programmes contre la violence, services de garde, aide juridique ou éducation, soutien aux organismes à but non lucratif, etc.

4. Certaines des conséquences des compressions budgétaires sont le travail précaire, le manque de personnel, l'accroissement de la charge de travail, le soutien financier minimal, la polyvalence des rôles, la fatigue, le stress, etc.

5. Voir Clément, Michèle, (1995), «Virage néolibéral, virage patriarcal: une entrevue avec Lucie Bélanger de Relais-Femmes», Service social, vol. 44, no 2, p. 10.

6. Voir le texte de Cardinal, Linda, «Femmes et francophonie : une relecture du rapport ethnicité-féminité» dans Andrew, C., L. Cardinal, F. Houle et G. Paquet (dir. de publ.) (1992). L'ethnicité à l'heure de la mondialisation, Ottawa, ACFAS-Outaouais.

7. Voir Corin, Ellen, Gilles Bibeau, Jean-Claude Martin et Robert Laplante (1990). Comprendre pour soigner autrement, Montréal, Presses de l'Université de Montréal.

8. NDLR: Trop souvent les femmes francophones vivant en milieu minoritaire ont acquiescé à un rôle de «francophones de service» pour justement se conformer à la rectitude politique.

9. Voir Lévesque, B. et L. Mager (1992). «Vers un nouveau contrat social? Éléments de problématique pour l'étude du régional et du social», dans J. L. Klein et C. Gagnon (dir. de publ.) Les partenaires du développement face au défi local, Chicoutimi, GRIR, Université du Québec à Chicoutimi.Voir aussi White, Deena (1990). «Le pouvoir de la culture: les aspects politiques de l'intervention en santé mentale», Santé, Culture, Health, vol. 7, 2-3.

10. Voir Jocelyne Lamoureux (1994). Le partenariat à l'épreuve, Montréal, Éditions SaintMartin, 1994: 185.

11. Ibid. : 185.

12. La coopération conflictuelle ou encore celle de participation contradictoire sont des expressions paradoxales servant à désigner le partenariat. Elles cherchent à indiquer 
que «Les interactions partenariales ne vont pas effectivement d'elles-mêmes. Elles se négocient serré, au pouce à pouce, ce qui implique, outre l'obligation de négocier, la nécessité d'amputer ou de partager son pouvoir, ses prérogatives ou ses innovations, la nécessité de céder des facettes de son identité spécifique, de se voir définir dans les termes de l'autre sans toujours s'imaginer qu'on y perd son essence» (Ibid. : 185-186). L'expression coopération conflictuelle a été explorée par B. Lévesque et L. Mager (1992) [voir note 5], par Doré, G. (1990). «L'enjeu de la «coopération conflictuelle» pour les groupes communautaires», conférence prononcée au colloque «Politiques économiques et politiques sociales : 18 mois après le libre-échange», Montréal, Université de Montréal, automne, et par Dommergues, P. (1988). La société du partenariat, Paris, Anthropos. 